\title{
The complete mitochondrial genome of a parthenogenetic ant Monomorium triviale (Hymenoptera: Formicidae)
}

\author{
Naoto Idogawa $^{a} \mathbb{D}$, Chih-Chi Lee $^{b} \mathbb{D}$, Chin-Cheng Scotty Yang ${ }^{c} \mathbb{D}$ and Shigeto Dobata ${ }^{d}$

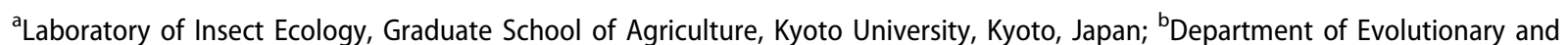 \\ Environmental Biology, Institute of Evolution, University of Haifa, Haifa, Israel; 'Department of Entomology, Virginia Polytechnic Institute and

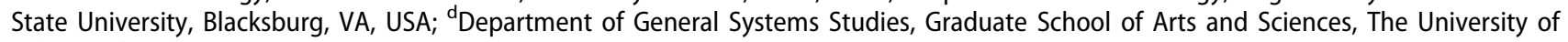 \\ Tokyo, Tokyo, Japan
}

\begin{abstract}
Monomorium is one of the most species-rich yet taxonomically problematic ant genus. An East Asian species, M. triviale Wheeler, W.M., 1906, is reproduced by obligate thelytokous parthenogenesis and performs strict reproductive division of labor. We sequenced the $M$. triviale mitogenome using next-generation sequencing methods. The circular mitogenome of $M$. triviale was $16,290 \mathrm{bp}$ in length, consisting of 13 protein-coding genes, two ribosomal RNA genes, 22 transfer RNAs, and a single noncoding region of $568 \mathrm{bp}$. The base composition was AT-biased (82\%). Gene order rearrangements were detected and likely to be unique to the genus Monomorium. We announce the $M$. triviale mitogenome as additional genomic resources for elucidating phylogenetic and taxonomic problems of Monomorium and comparative genomics of parthenogenetic ant species.
\end{abstract}

\section{ARTICLE HISTORY}

Received 3 March 2021

Accepted 15 August 2021

\section{KEYWORDS}

Hymenoptera; Formicidae; thelytoky;

Monomorium; mitogenome
In the hyperdiverse ant subfamily Myrmicinae, Monomorium Mayr, 1855 is one of the most species-rich genera with over 300 described species including several successful tramp species such as the flower ant M. floricola and M. salomonis, and the pharaoh ant M. pharaonis (Pontieri and Linksvayer 2019). However, recent studies (Ward 2015; Sparks 2019) suggest polyphyly of this genus, and genomic resources are therefore essential for resolving such a taxonomic issue.

An East Asian species, M. triviale Wheeler, W.M., 1906 is particularly of our interest as it reproduces by thelytokous parthenogenesis where virgin queens produce both workers and next-generation queens (Gotoh et al. 2012; Idogawa et al. 2021). To date, only partial mitochondrial DNA sequences have been reported, with all of which being identical among populations in Japan (Idogawa et al. 2021). Hence, a complete mitochondrial genome of this species can provide additional information for further analysis. Here, we present the first complete mitogenome for $M$. triviale.

A colony of $M$. triviale headed by a single queen was collected in Takaragaike Park, Kyoto, Japan (35.060087 N, 135.788488 E) on 9 September 2017. The queen and her parthenogenetic offspring produced later in the laboratory (larvae and pupae, approximately 100 individuals) were fixed in 99.5\% EtOH. We extracted genomic DNA from the pooled individuals with DNeasy Blood and Tissue kit (Qiagen, Hilden, Germany). We sequenced the pooled DNA on the HiSeq $X$ sequencer (Illumina, San Diego, CA) at Macrogen Japan Corp.
(Tokyo, Japan). After removing adapters with Trimmomatic v0.39 (Bolger et al. 2014), we conducted de novo mitogenome assembly based on 4,327,159 paired-end sequence reads using NOVOPlasty v3.6 (Dierckxsens et al. 2017), with Solenopsis invicta mitogenome (NCBI accession: NC_014672) as a seed. Average read coverage of the mitogenome assembly was 37,840, providing ample depth for correctness. We annotated protein-coding genes (PCGs), rRNAs, and tRNAs using GeSeq (Tillich et al. 2017), MITOS (Bernt et al. 2013), NCBI ORF-finder (Rombel et al. 2002), and ARWEN (Laslett and Canbäck 2008). The sequence information was deposited in the DNA Data Bank of Japan under accession number: LC605004. A specimen was deposited at the Laboratory of Insect Ecology, Graduate School of Agriculture, Kyoto University (http://www.insecteco.kais.kyoto-u.ac.jp; N. Idogawa; under the voucher number Mtri_20170909_4).

The complete mitogenome of $M$. triviale was $16,920 \mathrm{bp}$ in length, which is comparable to those of other ant species. The nucleotide composition was AT-biased (82\%). The mitogenome contained 13 PCGs, two rRNAs, and 22 tRNAs, which are typical for most metazoan animals. All PCGs used ATG or ATT as the start codon and TAA or TAG as the stop codon. The tRNAs, ranging in size from 59 to $74 \mathrm{bp}$, were similar to those of other ants (54-90 bp). The control region presumably corresponded to the single largest non-coding AT-rich region (569 bp, A+T $94 \%)$. Gene order of $M$. triviale was identical to that of a congener M. pharaonis (NC_051486.1). Notably, the gene order of

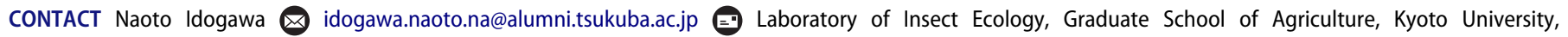
Kyoto, Japan

(C) 2021 The Author(s). Published by Informa UK Limited, trading as Taylor \& Francis Group.

This is an Open Access article distributed under the terms of the Creative Commons Attribution License (http://creativecommons.org/licenses/by/4.0/), which permits unrestricted use, distribution, and reproduction in any medium, provided the original work is properly cited. 


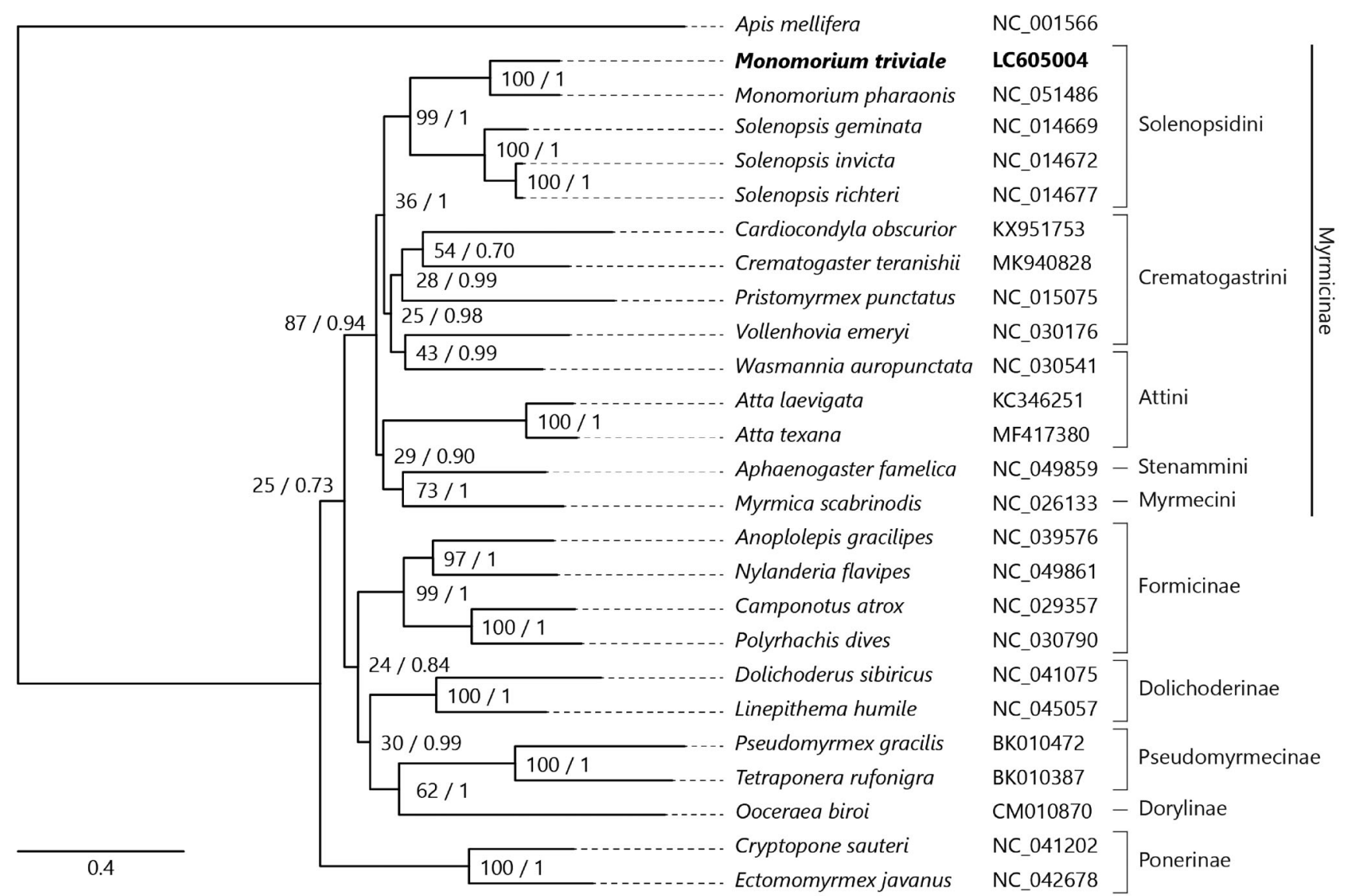

Figure 1. Maximum-likelihood (1000 bootstrap repeats) and Bayesian inference (100,000 generations) trees showing phylogenetic relationships among 25 ant species. A honeybee, Apis mellifera was used as an outgroup. Phylogenetic tree was drawn based on maximum-likelihood tree. Monomorium triviale in bold is the result obtained in this study. The numbers above branches indicate bootstrap support values for maximum-likelihood tree and posterior probability for Bayesian inference tree, in order.

the two Monomorium species had two gene rearrangements: an inversion between trnP and ND1 (Myrmicinae, ND6-CYTBtrnS; Monomorium, trnS-CYTB-ND6; underlines indicate inverted genes) and translocations between ND3 and trnF (Myrmicinae common, trnA-trnR-trnN-trnS-trnE; Monomorium, trnR-trnE-trnAtrnN-trnS). This feature was different from the common gene order of the subfamily Myrmicinae and likely unique to Monomorium ants (Babbucci et al. 2014; Park 2020). This may help identify the genus Monomorium sensu stricto, in addition to nucleotide substitutions.

We inferred the phylogenetic relationships of 25 ant species using the concatenated nucleotide sequences of all 13 PCGs, with the honeybee Apis mellifera as an outgroup. Sequence alignment was constructed using ClustalW (Thompson et al. 2003) implemented in MEGA-X (Kumar et al. 2018). The GTR $+I+G$ model was determined as a best-fit model by ModelTest-NG v0.1.6 (Darriba et al. 2020). Both a maximum-likelihood tree made by RAxML-NG v1.0.0 (Kozlov et al. 2019) and Bayesian inference tree made by MrBayes v3.2.7 (Ronquist et al. 2012) consistently support the current phylogenetic placement of Monomorium in the subfamily Myrmicinae (Figure 1).

In conclusion, the newly sequenced complete mitochondrial genome of $M$. triviale provides additional resources for further phylogenetic characterization of the taxonomically problematic genus Monomorium and comparative genomics of parthenogenetic ant species.

\section{Acknowledgements}

The authors are grateful to Kenji Matsuura who allowed us to use his laboratory.

\section{Disclosure statement}

No potential conflict of interest was reported by the author(s).

\section{Funding}

This work was supported by a Japan Society for the Promotion of Science (JSPS) Research Fellowship for Young Scientists to NI [19J22242] and a Grant from the Secom Science and Technology Foundation to SD.

\section{ORCID}

Naoto Idogawa (iD http://orcid.org/0000-0003-4055-8204

Chih-Chi Lee (ID) http://orcid.org/0000-0002-8778-1449

Chin-Cheng Scotty Yang (D) http://orcid.org/0000-0003-0967-5170

Shigeto Dobata (iD http://orcid.org/0000-0003-1586-6758

\section{Data availability statement}

The genome sequence data that support the findings of this study are openly available in DDBJ/GenBank at https://www.ddbj.nig.ac.jp under the accession no. LC605004. The associated BioProject, SRA, and BioSample numbers are PRJDB12079, DRX301164, and SAMD00394597, respectively. 


\section{References}

Babbucci M, Basso A, Scupola A, Patarnello T, Negrisolo E. 2014. Is it an ant or a butterfly? Convergent evolution in the mitochondrial gene order of Hymenoptera and Lepidoptera. Genome Biol Evol. 6(12):3326-3343.

Bernt M, Donath A, Jühling F, Externbrink F, Florentz C, Fritzsch G, Pütz J, Middendorf M, Stadler PF. 2013. MITOS: improved de novo metazoan mitochondrial genome annotation. Mol Phylogenet Evol. 69(2):313-319.

Bolger AM, Lohse M, Usadel B. 2014. Trimmomatic: a flexible trimmer for Illumina sequence data. Bioinformatics. 30(15):2114-2120.

Darriba D, Posada D, Kozlov AM, Stamatakis A, Morel B, Flouri T. 2020. ModelTest-NG: a new and scalable tool for the selection of DNA and protein evolutionary models. Mol Biol Evol. 37(1):291-294.

Dierckxsens N, Mardulyn P, Smits G. 2017. NOVOPlasty: de novo assembly of organelle genomes from whole genome data. Nucleic Acids Res. 45(4):e18.

Gotoh A, Billen J, Tsuji K, Sasaki T, Ito F. 2012. Histological study of the spermatheca in three thelytokous parthenogenetic ant species, Pristomyrmex punctatus, Pyramica membranifera and Monomorium triviale (Hymenoptera: Formicidae). Acta Zool. 93(2):200-207.

Idogawa N, Sasaki T, Tsuji K, Dobata S. 2021. Comprehensive analysis of male-free reproduction in Monomorium triviale (Formicidae: Myrmicinae). PLOS One. 16(4):e0246710.

Kozlov AM, Darriba D, Flouri T, Morel B, Stamatakis A. 2019. RAxML-NG: a fast, scalable and user-friendly tool for maximum likelihood phylogenetic inference. Bioinformatics. 35(21):4453-4455.

Kumar S, Stecher G, Li M, Knyaz C, Tamura K. 2018. MEGA X: molecular evolutionary genetics analysis across computing platforms. Mol Biol Evol. 35(6):1547-1549.
Laslett D, Canbäck B. 2008. ARWEN: a program to detect tRNA genes in metazoan mitochondrial nucleotide sequences. Bioinformatics. 24(2): 172-175.

Park J, Xi H, Park J. 2020. The complete mitochondrial genome of Aphaenogaster famelica (Smith, 1874) (Hymenoptera: Formicidae). Mitochondrial DNA B Resour. 5(1):492-494.

Pontieri L, Linksvayer TA. 2019. Monomorium. In: Starr C. (ed.), Encyclopedia of social insects. Cham: Springer International Publishing; p. 1-6.

Rombel IT, Sykes KF, Rayner S, Johnston SA. 2002. ORF-FINDER: a vector for high-throughput gene identification. Gene. 282(1-2): 33-41.

Ronquist $F$, Teslenko $M$, van der Mark $P$, Ayres DL, Darling $A$, Höhna $S$, Larget B, Liu L, Suchard MA, Huelsenbeck JP, et al. 2012. MrBayes 3.2: efficient Bayesian phylogenetic inference and model choice across a large model space. Syst Biol. 61(3):539-542.

Sparks KS, Andersen AN, Austin AD. 2019. A multi-gene phylogeny of Australian Monomorium Mayr (Hymenoptera: Formicidae) results in reinterpretation of the genus and resurrection of Chelaner Emery. Invertebr Syst. 33:225-236.

Thompson JD, Gibson TJ, Higgins DG. 2003. Multiple sequence alignment using ClustalW and ClustalX. Curr Protoc Bioinformatics.

Tillich M, Lehwark P, Pellizzer T, Ulbricht-Jones ES, Fischer A, Bock R, Greiner S. 2017. GeSeq- versatile and accurate annotation of organelle genomes. Nucleic Acids Res. 45(W1):W6-W11.

Ward PS, Brady SG, Fisher BL, Schultz TR. 2015. The evolution of myrmicine ants: phylogeny and biogeography of a hyperdiverse ant clade (Hymenoptera: Formicidae). Syst Entomol. 40(1):61-81. 